\title{
Serial liver biopsies in blood donors with persistent $\mathrm{HB}_{\mathrm{s}}$ antigenaemia
}

\author{
E. TAPP, D. M. JONES, D. HOLLANDERS, AND I. W. DYMOCK 1 \\ From the Departments of Pathology and Medicine, Withington Hospital, Manchester
}

SYNOPSIS Initial liver biopsies from asymptomatic $\mathrm{HB}_{\mathrm{S}}$ antigen positive blood donors showed $\mathrm{a}_{\bar{\omega}}$ range of histological abnormalities ranging from minor parenchymal lesions to cirrhosis. Twenty of these have now been followed up for periods of up to four years and during that time have had ato least two liver biopsies. Throughout the period of study all the donors have remained carriers of 0 $\mathrm{HB}_{\mathrm{S}} \mathrm{Ag}$, and there was no significant variation in the titres of antigen or the electron microscopic $\overrightarrow{0}$ appearances of the serum in individual donors. The histological appearances of subsequent liverio biopsies were not always the same as those seen initially, and while there appeared to be some $e^{+}$ improvement in three cases, there were nine in which the histological appearances were worse.

Over the past few years there have been a number of reports of liver disease in asymptomatic blood donors with persistent hepatitis $B_{S}$ antigenaemia. The incidence and severity of liver disease revealed by these studies probably depends to some extent on the population from which they are drawn. In Copenhagen only a small proportion of donors were found to have histological evidence of liver disease (Banke et al, 1971; Reinicke et al, 1972) but a high incidence of abnormalities has been reported from Denver (Singleton et al, 1971) and from Chicago (Vittal et al, 1974). Bollin and his co-workers (1973) have suggested that drug addiction is an important factor influencing the presence of significant liver disease in the different centres. However, there were no narcotic users in the series from north-west England (Woolf et al, 1974) and yet a high incidence of histological abnormalities, including cirrhosis and chronic aggressive hepatitis, was found.

It is clearly important to determine the eventual outcome of the disease in these asymptomatic donors. This paper describes the findings in 20 of them who have had at least two liver biopsies with a minimal interval of 12 months between them.

\section{Patients and methods}

The original group of 34 blood donors described by Woolf et al (1974) was increased in number by the addition of further cases from the screening proce-

${ }^{1}$ Present address: Department of Gastroenterology, Stepping Hill Hospital, Stockport

Received for publication 2 March 1976 dure, and from this enlarged group 20 donors haveep had liver biopsies on at least two occasions during the follow-up period. In addition, routine liver function tests have been repeated at three-mont intervals, and most of the donors have had at least two bromsulphalein retention tests. Tests $\mathrm{HB}_{\mathrm{S}} \mathrm{Ag}$ and determinations of the titre of antigen have been repeated during the period of surveillances using a haemagglutination technique (Burrough\$ Wellcome). The distribution of the types of antigen particles in the serum on electron microscopy was. evaluated on a serum sample obtained at the time of the initial liver biopsy ('first' sample) and also at the time of the most recent biopsy ('second' sample).

\section{Results}

The mean age of the group was $36 \cdot 8$ (range 20-55) years. Sixteen were male and four were female.

HEPATITIS B ANTIGEN IN THE SERUM All the donors remained carriers of $H_{B} A g$ through out the study, and no significant differences in titre were detectable in individual donors during this period. Similarly, no significant differences were seen between the electron microscopic appearances of thew 'first' and 'second' samples. In those that had Dane particles initially, these were still demonstrable in the 'second' sample, and there were no changes in the relative proportions of these and other particle types?

HISTOLOGY

The original and subsequent histological diagnoses 
in the donors are shown in the table. In addition the following points are of note.

1 Cirrhosis The original biopsy with cirrhosis showed a marked inflammatory infiltrate in the parenchyma and piecemeal necrosis. In a subsequent biopsy two years later the inflammation had subsided, the appearances being those of cryptogenic cirrhosis.

2 Chronic aggressive hepatitis In both subjects who subsequently showed cirrhosis, there was a diminution in the amount of inflammation in the portal tracts. Three cases remained within this group; the inflammation in the portal tracts was unchanged in one, decreased in one, and increased in the other.

3 Chronic persistent hepatitis of the six cases of chronic persistent hepatitis which remained within this group there were two in which the inflammation in the portal tracts had diminished in intensity.

\section{CLINICAL COURSE}

Only one donor has developed symptoms during the follow-up period. This 26-year-old woman had abnormal liver function tests when first seen. The initial liver biopsy showed a chronic aggressive hepatitis. She subsequently developed symptoms in keeping with the clinical syndrome of chronic active hepatitis and was treated with corticosteroids and azathioprine. With this her symptoms improved and biochemical tests became normal. She remains well four years after presentation and the antigen titre is unaltered. The repeat liver biopsy showed a cirrhosis with aggressive features.

\section{BIOCHEMISTRY}

With the exception of the patient described above, there were no significant changes in the routine liver function tests or in the bromsulphalein retention tests in the carriers during the period of this investigation.

\begin{tabular}{llllll}
\hline Initial biopsy & \multicolumn{5}{l}{ Most recent biopsy } \\
\cline { 2 - 5 } & Cirrhosis & CAH & CPH & FPN \\
\hline $\begin{array}{l}\text { Cirrhosis } \\
\begin{array}{c}\text { Chronic aggressive } \\
\text { hepatitis }\end{array}\end{array}$ & 1 & 1 & 0 & 0 & 0 \\
$\begin{array}{c}\text { Chronic persistent } \\
\text { hepatitis }\end{array}$ & 8 & 2 & 3 & 0 & 0 \\
$\begin{array}{c}\text { Focal parenchymal } \\
\text { necrosis }\end{array}$ & 6 & 0 & 2 & 6 & 0 \\
\hline
\end{tabular}

Table $A$ comparison of histological diagnoses in the initial and most recent biopsies

\section{Discussion}

Our results show that the histological appearances of subsequent biopsies are not always the same as those seen initially, and while there appeared to be some improvement in three cases there were nine in which the histological appearances were worse. However, a good deal of caution should be exercised in considering whether there is sufficient evidence from these biopsies to say that the liver disease has progressed. The problem of sampling error has to be constantly borne in mind when one is examining small biopsies from a large organ. The chance of missing cirrhosis is probably minimal. Scheuer (1970), in a review of the use of liver biopsy in the diagnosis of cirrhosis, quoted the high degree of accuracy reported by Braunstein (1956) and Baggenstoss (1966). Both these authors used the Vim Silverman needle, which has a similar cutting action to that used in the present series and is preferable to the Menghini needle which may glance off hard tissue and so selectively sample the softer parenchyma. The differentiation of chronic aggressive and chronic persistent hepatitis is more difficult, and here sampling error could be relevant. There is no doubt that the severity of the disease process varies in different parts of the liver, and Dudley et al (1972) reported that one of their patients had areas in the liver which were consistent with chronic persistent and chronic aggressive hepatitis in different parts of the same specimen.

Although there appeared to be a deterioration of the histological appearances in some carriers from focal parenchymal necrosis to chronic persistent hepatitis, in some from chronic persistent hepatitis to chronic aggressive hepatitis, and in others from chronic aggressive hepatitis to cirrhosis, in no one individual had the histological appearances progressed through more than one of these stages. More prolonged observation of this type of carrier will be necessary if the possibility of a continuous progression from focal parenchymal necrosis to cirrhosis is to be demonstrated. The eventual diminution in the inflammatory activity in the liver may leave the histological appearance of cryptogenic cirrhosis.

The biochemical investigations do not show any corresponding evidence of deterioration in the routine liver function tests or the bromsulphalein retention test. These are relatively insensitive indicators of liver disease in asymptomatic blood donors and are only persistently abnormal in cases of chronic aggressive hepatitis (Woolf et al, 1974).

The titres of antigen in individual carriers remained constant over the period of study and the electron microscopic appearance of the serum remained unchanged. Previously the titre of antigen has been shown to be unrelated to the histological appearance 
of the liver (Woolf et al, 1974). In this study neither the titre nor the presence of Dane particles could be correlated with the progression of disease.

\section{References}

Baggenstoss, A. H. (1966). Morphologic and etiologic diagnoses from hepatic biopsies without clinical data. Medicine (Baltimore), 45, 435-443.

Banke, O., Dybkjaer, E., Nordenfelt, E., and Reinicke, V. (1971). Australia antigen and antibody in 10,000 Danish blood-donors. (Letter.) Lancet, 1, 860-861.

Bollin, T. D., Davis, A. E., and Liddelow, A. G. (1973). Liver disease and cell-mediated immunity in hepatitis-associated antigen (HAA) carriers. Gut, 14, 365-368.

Braunstein, H. (1956). Needle-biopsy of the liver in cirrhosis. Arch. Path., 62, 87-95.

Dudley, F. J., Scheuer, P. J., and Sherlock, S. (1972). Natural history of hepatitis-associated antigen-positive chronic liver disease. Lancet, 2, 1388-1393.

Reinicke, V., Dybkjaer, E., Poulsen, H., Banke, O., Lyllof $\stackrel{\varrho}{=}$ K., and Nordenfelt, E. (1972). A study of Australia-anti-으 gen-positive blood donors and their recipients, with special reference to liver histology. New Engl. J. Med., 286, 867870.

Scheuer, P. J. (1970). Liver biopsy in the diagnosis of cirrhosis. Gut, 11, 275-278.

Singleton, J. W., Fitch, R. A., Merrill, D. A., Kohler, P. F., $\overline{\overline{\mathcal{C}}}$ and Rettburg, W. A. H. (1971). Liver disease in Australia- $\overparen{D}$ antigen-positive blood-donors. Lancet, 2, 785-787.

Vittal, S. B. V., Dourdourekas, D., Shobassy, N., Gerber, M., ֶొ Telischi, M., Szanto, P. B., Steigmann, F., and Clowdus, B. F. (1974). Asymptomatic hepatic disease in blood donors. with hepatitis B antigenemia. Amer.J. clin. Path., 62, 649- $\overrightarrow{-}$ 654.

Woolf, I. L.., Boyes, B. E., Jones, D. M., Whittaker, J. S., Tapp, E., MacSween, R. N. M., Renton, P. H., Stratton, 응 F., and Dymock, I. W. (1974). Asymptomatic liver disease î in hepatitis B antigen carriers. J. clin. Path., 27, 348-352. 